\title{
Für mehr Chancengerechtigkeit an Schweizer Gymnasien? Ein Projektbericht über die Initiative "Go for it!"
}

* itta.baver@geo.uzh.ch, Institut für Geographie, Universität Zürich

Der Beitrag stellt eine private Initiative vor, die sozial benachteiligten Schülerinnen und Schülern kostenlose Vorbereitungskurse für die Aufnahmeprüfung ans Gymnasium in einem Deutschschweizer Kanton anbietet.

Keywords: Soziale Selektivität, Bildung, Schweiz, Jugendliche

More equality at Swiss Grammar Schools? A project-report of the initiative "Go for it!"

This paper presents a private initiative which offers free of charge preparation-courses to socially disadvantaged students, who want to sit the central entrance examination to attend Gymnasium (Grammar School) in a German-speaking canton in Switzerland.

Keywords: social selectivity, education, Switzerland, young people

\section{$1 \quad$ Einleitung}

Eine Gruppe Jugendlicher zwischen 12 und 13 Jahren kommt jeden Mittwochnachmittag und jeden zweiten Samstagvormittag für sechs Monate in einem Zürcher Gemeinschaftsraum zusammen, um freiwillig mit zwei Lehrpersonen Deutsch und Mathematik zu lernen. Sie konstruieren Mittelsenkrechten, lösen gemeinsam knifflige Mathe-Aufgaben zur Proportionalität, beschäftigen sich mit Wortarten oder besprechen Jugendbücher und griechische Sagen.

All das steht üblicherweise nicht unbedingt auf der Wunschliste von Jugendlichen für einen schulfreien Mittwochnachmittag oder einen Samstagvormittag. Warum kommen diese Jugendlichen regelmäßig zusammen, obwohl sie doch alle gute bis sehr gute Noten in der Schule haben? Leyla, Serkan, Aylül, Denis, Ananthukama und ihre Kolleginnen und Kollegen stecken sehr viel Zeit und Energie in den Gymi-Vorbereitungskurs des Vereins „Go for it!" ${ }^{\text {11 }}$ und wissen doch nicht, ob sie den Sprung aufs Gymnasium tatsächlich schaffen. Der Verein „Go for it!“ ist eine private Initiative, die sozial benachteiligten Jugendlichen einen

\footnotetext{
Um die Identität der Jugendlichen zu schützen wurden ihre Namen anonymisiert. Auch die Bezeichnung der Initiative „Go for it!“ wurde entsprechend umbenannt. Die Quellen mit lokal identifizierbarem Bezug können auf Anfrage offengelegt werden.
}

kostenlosen Gymi-Vorbereitungskurs anbietet. Denn nur wer gute Noten in der 6 . Klasse und ein genügendes Prüfungsergebnis in der Zentralen Aufnahmeprüfung hat, wird in ein Langzeitgymnasium in diesem Kanton der Deutschschweiz aufgenommen. Und das ist nur ein kleiner Bruchteil aller Schüler/innen der 6. Jahrgangsstufe, die zumeist aus gut situierten, bildungsnahen Familien kommen. Was aber bedeutet der Übertritt aufs Gymnasium für Jugendliche aus einkommensschwachen Familien? Was treibt Jugendliche wie Leyla, Serkan, Aylül, Denis und Ananthukama an, sich ein halbes Jahr im Kurs des Vereins „Go for it!" auf die Aufnahmeprüfung vorzubereiten und die Prüfung abzulegen, trotz ihrer eher ungünstigen Aussichten auf Erfolg? Diesen und anderen Fragen geht die Autorin zusammen mit Dr. Sara Landolt (Universität Zürich) im Rahmen der wissenschaftlichen Begleitung des Bildungsprojektes seit drei Jahren nach.

\section{Der lokale Bildungskontext}

Um die Beweggründe dieser Jugendlichen besser nachvollziehen zu können, hilft ein Blick in aktuelle Trends der Schweizer Bildungslandschaft. Die steigenden Qualifikationsanforderungen auf dem internationalen Berufsmarkt beeinflussen einerseits die Gestaltung von Bildungs- und Qualifikationsstruktu- 
ren auf nationaler und regionaler Ebene; andererseits wirkt sich der Trend zu höheren „Bildungsaspirationen" (Gresch 2012) in weiten Teilen der Gesellschaft auch darauf aus, welche Wünsche, Vorstellungen und Erwartungen an Kinder und Jugendliche in Bezug auf ihre Bildungsbiographien herangetragen werden. Wie junge Menschen diesen gestiegenen Anforderungen begegnen und welche Auswirkungen der zunehmende Leistungsdruck auf ihre Gesundheit, Freizeitgestaltung und Zukunftsvisionen hat, zeigen u.a. Studien von Inge Seiffge-Krenke (2006), die Bewältigungsstrategien von Jugendlichen in mehreren europäischen Ländern untersuchte und dabei Stresssituationen analysierte, die mehrheitlich von den schulischen Anforderungen und den Leistungsanforderungen der Jugendlichen an sich selbst ausgingen. Die Eltern nahmen dabei zum größten Teil eine positiv-unterstützende Rolle für die Jugendlichen ein. In einer groß angelegten Studie mit über 1500 Schweizer Jugendlichen legte die Juvenir 4.0-Studie Zahlen zum subjektiv empfundenen Leistungsdruck und Stress aus Sicht von jungen Menschen vor. Für etwa die Hälfte der Jugendlichen (46\%) sind Stress, Leistungsdruck und Überforderung keine Ausnahmesituationen, sondern gehören zu ihren Alltagserfahrungen. Als Ursache für die Belastungssituation werden vor allem Schule, Ausbildung, Uni und Beruf von 56\% der Jugendlichen angeführt, wobei sich die Mehrheit der Jugendlichen vor allen Dingen selbst unter großen Leistungsdruck setzt (Knittel et al. 2015). Zu einer ähnlichen Diagnose kommt der Kinder- und Jugendpsychiater Michael Schulte-Markwort in seiner Publikation mit dem provokativen Titel „Burn out kids - Wie das Prinzip Leistung unsere Kinder überfordert" (2015). Die Häufigkeit von Erschöpfungsdepressionen und Burnouts bei Jugendlichen hat in den letzten Jahren über alle sozialen Schichten hinweg drastisch zugenommen. Er fordert daher ein gesellschaftliches Umdenken, das Alternativen anbietet zum vorherrschenden Leistungsprinzip - im Interesse unserer Kinder. Weil ein gesellschaftliches Umdenken leichter eingefordert als umgesetzt werden kann, liegt es derzeit wohl an den Eltern, Lehrpersonen und Bezugspersonen von Kindern und Jugendlichen ihnen zuzuhören, ihren Selbstwert zu stärken, humanitäre Werte zu vermitteln und Handlungsalternativen aufzuzeigen, die innerhalb des gesellschaftlichen Systems auch Freiheiten und Eigenheiten erlauben.

Obwohl der Arbeitsmarkt in der Schweiz im europäischen Vergleich, gerade auch für Jugendliche, recht stabile Berufsaussichten verspricht und die Jugendarbeitslosigkeit (2019) mit 2,1\% auf einem sehr geringen Niveau ${ }^{2}$ liegt, geht der Trend auch hier zu höheren

https://www.amstat.ch/v2/index.jsp, 02.05.2019
Bildungsabschlüssen. Im Bildungsbericht der Schweiz wird prognostiziert, dass der Anteil der Bevölkerung mit einem tertiären Bildungsabschluss von rund $40 \%$ im Jahr 2015 auf ca. 60 \% im Jahr 2045 ansteigen wird (SKBF 2018). Tertiäre Bildungsqualifikationen (d.h. Hochschul- oder Fachhochschulabschlüsse) werden immer mehr zur Standardvoraussetzung für eine erfolgreiche Bildungs- und Berufsbiographie. Die föderale Bildungspolitik der Schweiz trug ihren Teil dazu bei, dass sich Bildungsverantwortung und entsprechende Schulstrukturen regional sehr unterschiedlich ausgebildet haben. Ein Musterbeispiel hierfür sind etwa die gymnasialen Maturitätsquoten, die zwischen den Kantonen stark variieren: Genf (29,4\%), Tessin (27,3\%), Zürich (20,0\%) und Thurgau (14,1\%) (Bezugsjahr 2016; BFS 20183).

In den letzten Jahrzehnten sind sowohl die $\mathrm{Zu}$ gangszahlen an das Gymnasium als auch die Maturitätsquoten in der Schweiz im nationalen Durchschnitt deutlich angestiegen (Oelkers 2008; SKBF 2014, 2018). Im Schnitt erwerben $20 \%$ eines Jahrgangs eine gymnasiale Maturität, was im Vergleich zu anderen OECD-Ländern eher sehr niedrig ist (Schweizer Kommission für Bildungsforschung 2018). An dieser Stelle ist es wichtig zu ergänzen, dass sich das Schweizer Bildungssystem durch eine Vielzahl an Möglichkeiten auszeichnet, die zu einem Bildungsabschluss mit Fach- und Hochschulzugang führen. Somit wird es nachvollziehbar, dass 2017 47,2\% der erwachsenen Schweizer und 37,9\% der erwachsenen Schweizerinnen (25-64 Jahre; ständiger Wohnsitz Schweiz) über einen Bildungsstand auf Tertiärstufe (höhere Berufsbildung oder Hochschule) verfügen (Bundesamt für Statistik 20194).

Eine besondere Situation innerhalb des sehr heterogenen Schweizer Bildungssystems stellt das Beispiel einer großen Stadt in der deutschsprachigen Schweiz dar, das hier im Mittelpunkt steht. Der Anteil der erwachsenen Bevölkerung, der über eine tertiäre Ausbildung verfügt, beträgt in dieser Stadt $47 \%$ (Quelle auf Anfrage). Familien aus diesen bildungsaffinen Schichten erhoffen sich für ihre Kinder zumeist ebenfalls einen möglichst hohen Bildungsweg, der über das Gymnasium zur Fachhochschule oder Hochschule führt. Dem gegenüber stehen Familien aus weniger sozial privilegierten Milieus und Migrationskontexten, die sich an die Hoffnung klammern den sozialen Aufstieg über eine erfolgreiche Bildungsbiographie ihrer Kinder zu schaffen (vgl. Fend 2009). Nicht selten verfügen die Eltern selbst über einen recht hohen

\footnotetext{
https://www.bfs.admin.ch/bfs/de/home/statistiken/katalogedatenbanken/tabellen.assetdetail.2421478.html 05.08.2019

4 https://www.bfs.admin.ch/bfs/de/home/statistiken/wirtschaftliche-soziale-situation-bevoelkerung/gleichstellung-frau-mann/ bildung/bildungsstand.html 05.08.2019
} 
Bildungsabschluss in ihrem Herkunftsland, der ihnen jedoch in der Schweiz formal nicht anerkannt wird und sie daher in weniger qualifizierten Arbeitsfeldern erwerbstätig sind (Baghdadi \& Riaño 2011; Landolt \& Thieme 2018). Hier stehen sich also die Angst vor dem gesellschaftlichen Abstieg oder das „Statuserhaltsmotiv" (Breen \& Golthorpe 1997) und die Hoffnung auf einen gesellschaftlichen Aufstieg über Bildungserfolg (Fend 2009) auf kleinstem Raum diametral entgegen. Diese Bildungsaspirationen werden weiter verstärkt durch eine lokale Bildungspolitik, die einerseits an einer strengen Reglementierung von Plätzen im Gymnasium über eine politisch gewünschte gymnasiale Übertrittsquote von rund $20 \%$ eines Jahrgangs festhält; andererseits den Aufbau von zwei neuen Gymnasien in der Agglomeration maßvoll vorantreibt, um den enormen Druck auf die städtischen Gymnasien bei stetig steigenden Schüler/ innenzahlen auch langfristig besser abzufedern (vgl. Bauer 2018).

Wie in vielen anderen Schweizer Kantonen gibt es im lokalen Fallbeispiel zwei Varianten des Gymnasiums - das Langzeitgymnasium (Jahrgangsstufen 7-12) und das Kurzzeitgymnasium (Jahrgangsstufen 9-12) - die beide zum gleichen Schulabschluss führen: die gymnasiale Maturität. Der Zugang zu beiden Gymnasien wird kantonal über ein selektives Aufnahmeverfahren geregelt. Dabei bestätigen sowohl die kantonalen Anmeldezahlen für die Aufnahmeprüfung ans Langzeitgymnasium (4194 angemeldete Schüler/ innen im Prüfungsjahr 2019) sowie die besseren Bestehensraten an der ersten Schwelle $(50.3 \%$ der angemeldeten Schüler/innen bestanden die Prüfung im Jahr 2019) einen klaren Trend hin zum Langzeitgymnasium. Für den Aufnahmetest ans Kurzzeitgymnasium meldeten sich im Jahr 2019, 3466 Jugendliche an, von denen aber nur 39.7\% erfolgreich waren (Quelle auf Anfrage).

Das Nadelöhr zum Langzeitgymnasium führt über ein selektives Aufnahmeverfahren, in das die Halbjahresnoten in den Fächern Deutsch und Mathematik (Jahrgangsstufe 6) ebenso eingehen wie die Ergebnisse aus der Zentralen Aufnahmeprüfung in Deutsch und Mathematik. Das Aufnahmeverfahren für das Kurzzeitgymnasium wird mittlerweile vergleichbar geregelt, über Vornoten und Aufnahmeprüfung. In einer Studie arbeiteten Moser et al. (2011) heraus, dass nicht nur die überwiegende Mehrheit der Schüler/innen an kantonalen Langzeitgymnasien Schweizer/innen sind (Für $81 \%$ der Schüler/innen ist Deutsch die Erstsprache), sondern auch, dass $85 \%$ der Gymnasiastinnen und Gymnasiasten aus privilegierten und eher privilegierten Familien stammen (Moser et al. 2011: 75-76). Die Chancen für einen erfolgreichen Übertritt ins Gymnasium wird für Kin- der aus bildungsfernen Familien weiterhin erschwert durch den Zusammenhang von sehr guten Zeugnisnoten von Kindern aus privilegierten Familien, die zusätzlich noch private Angebote zur Prüfungsvorbereitung in Anspruch nehmen, um ihre Bestehenschancen in der Prüfung weiter zu erhöhen (Moser et al. 2011: 77). Der Übertritt ins Langzeitgymnasium kennzeichnet in der aktuellen Form eine besonders neuralgische Stelle im Schweizer Bildungssystem, an dem sich die soziale Selektivität von Bildungschancen und Bildungswegen von Kindern besonders deutlich zeigt (Moser et al. 2011: 82).

Die Situation auf dem lokalen Bildungsmarkt an der Schwelle zum Gymnasium (v. a. Langzeitgymnasium) lässt sich folgendermaßen zusammenfassen:

- Viele Schülerinnen und Schüler zeigen am Ende der Primarschule eine hohe Leistungsbereitschaft und stehen unter einem hohen Erfolgsdruck. Dies äußert sich u.a. dadurch, dass sich über 4000 Schüler/innen der Zentralen Aufnahmeprüfung für das Langzeitgymnasium stellen und nur etwa die Hälfte Erfolg hat.

- Der Trend zu höherer Bildung am Gymnasium hat den Druck auf das Langzeitgymnasium intensiviert.

- Die gestiegenen Bildungsaspirationen in der Mehrheitsgesellschaft schufen die Voraussetzungen dafür, dass sich im urbanen Gebiet der Fallstudie, vor allem um die Vorbereitung der Zentralen Aufnahmeprüfung, ein dynamischer Bildungsmarkt entwickelte. Es ist ein sehr heterogenes Feld an Akteuren und Akteurinnen und Angeboten, die diesen Markt aktiv gestalten: privat-profitorientierte Lernstudios, private Initiativen (non-profit), schulische Vorbereitungskurse (non-profit) und informelle Netzwerke unter Familien und Schülerinnen und Schülern.

\section{Die Initiative „Go for it!“}

Die eingangs erwähnten Jugendlichen Leyla, Serkan, Aylül, Denis, Ananthukama und ihre Kolleginnen und Kollegen besuchen den Gymi-Vorbereitungskurs des gemeinnützigen Vereins „Go for it!”. Der Verein hat sich zum Ziel gesetzt, talentierte und leistungsbereite Kinder, mit und ohne Migrationshintergrund, aus sozial benachteiligten Familien dabei zu unterstützen, sich bestmöglich auf das selektive Übertrittsverfahren ans Gymnasium vorzubereiten. Die Forderung nach mehr Chancengerechtigkeit von Kindern aus einkommensschwachen, aber bildungsaffinen Familien erhält somit ein konkretes Gesicht und kostenneutrales Förderangebot. Obwohl der Vorbereitungskurs grundsätzlich allen offen steht, ist 
die Teilnehmer/innenzahl auf 13 Kinder beschränkt, damit diese Gruppe an ausgewählten Kindern optimal auf die Aufnahmeprüfung vorbereitet werden kann. Einen begehrten Platz zu ergattern, ist nicht einfach. Jede/r Bewerber/in muss ein umfangreiches Dossier vorlegen, das folgende Unterlagen enthält: ein ausgefülltes Anmeldeformular für den Kurs, ein Empfehlungsschreiben der Primarschullehrperson, ein Nachweis über die letzte Steuererklärung beider Elternteile sowie das Zwischenzeugnis aus der 5. Jahrgangsstufe. Kandidatinnen und Kandidaten, die die Aufnahmekriterien erfüllen, werden vom Verein zu einer schriftlichen Textproduktion (z. B. offene Fragen zur Schule und Hausaufgaben, Motivation für Kursbesuch) und einem Gespräch mit zwei Vereinsmitgliedern eingeladen. Auf der Basis dieser Unterlagen und des Gesamteindrucks entscheidet der Verein welche Schüler/innen in den Kurs aufgenommen werden.

Den ersten Förderkurs besuchten im Schuljahr 2016/17 sieben Mädchen und sechs Jungen. Für elf von ihnen war nicht Deutsch die Erstsprache, sondern Albanisch (1), Arabisch (1), Kroatisch (1), Mazedonisch (1), Serbisch (2), Spanisch (1), Tamilisch (3) und Türkisch (1). Drei Schüler/innen bestanden die Aufnahmeprüfung, aber nur eine Schülerin wurde nach der Probezeit definitiv an eine Kantonsschule aufgenommen.

Der zweite Förderkurs wurde von sechs Mädchen und sieben Jungen im Schuljahr 2017/18 besucht. Nur für eine war Deutsch ihre Erstsprache. Acht Kinder waren an der Aufnahmeprüfung erfolgreich und sieben haben die Probezeit am Gymnasium mit Erfolg bestanden.

Im dritten Förderkurs wurden acht Mädchen und fünf Jungen im Schuljahr 2018/19 aufgenommen, für die ausnahmslos Deutsch nicht ihre Erstsprache war. Vier Kinder bestanden die Aufnahmeprüfung und bereiten sich mit zwei nicht erfolgreichen Kursbesucher/ innen (die in eine Sekundarschule, höchstes Niveau, wechseln) im zweiten Kurshalbjahr gemeinsam auf den Übertritt in die Sekundarstufe I vor. Neben schulischen Inhalten, v. a. Deutsch und Mathematik, lesen und diskutieren die Kinder weiterhin zusammen Jugendbücher und besprechen wie sie am besten das Lernen lernen. Am letzten Tag des Kurses unternehmen die Kinder mit ihren zwei Mentoren (Lehrpersonen für Deutsch und Mathematik) einen Ausflug an eine Hochschule und gehen zusammen noch ein Eis essen. Während der Probezeit am Gymnasium (1. Schulhalbjahr) wird vom Verein zudem ein Unterstützungsprogramm an einer ausgewählten Kantonsschule finanziert, das jeder/m „Go for it!“-Schüler/in eine/n Tutor/in zur Seite stellt, den/die die Jugendlichen je nach Bedarf und Wunsch kontaktieren und um Hilfe (z. B. bei den Hausaufgaben, Prüfungsvorbereitungen oder bei allgemeinen Fragen zur Schule) bitten können. Dadurch soll sichergestellt werden, dass die Schüler/innen während der Probezeit, die zwischen $10 \%$ und $20 \%$ der Siebtklässler/innen nicht bestehen und auf eine Sekundarschule verwiesen werden, auf ein Netzwerk zurückgreifen können, das sie langfristig fördert und unterstützt.

\section{4 (Selbst-)Kritische Töne einer sozial enga- gierten Jugend- und Bildungsgeographie}

Als wir dem Verein vor drei Jahren anboten die wissenschaftliche Begleitung des Projekts zu übernehmen, war es uns wichtig herauszustellen, dass wir diese Aufgabe im Sinne einer sozial engagierten Bildungsgeographie übernähmen, der aufgrund unserer beruflichen Verpflichtungen enge zeitliche Grenzen gesetzt sein würden. Mittlerweile haben wir zwei der bisher drei Kurs-Generationen kennengelernt und haben die verschiedenen Phasen des Projekts - von der Auswahlphase der Kandidatinnen und Kandidaten, über die intensive Prüfungsvorbereitung, die anschließende Kursgestaltung nach der Prüfung sowie die Probezeit am Gymnasium - mit teilnehmender Beobachtung, Leitfadeninterviews (mit Kindern, Eltern, Vorstandsmitgliedern, Deutsch-Mentor und Mathematik-Mentorin, Primarschullehrpersonen) und in Vorstandspräsentationen dokumentiert und analysiert. Obschon wir in alle Phasen des Projekts mittlerweile einen guten Einblick haben, mussten wir feststellen, dass die wissenschaftliche Begleitung für uns als qualitativ forschende Sozialgeographinnen eine persönliche Herausforderung ist. Einerseits möchten wir den Kontakt zu den Jugendlichen intensivieren, um genauere Aussagen machen zu können, wie sie ihre eigene Entwicklung während und nach dem Kursbesuch einschätzen und wie sich ihre schulischen Erfahrungen auf ihre Familie und ihren Freundeskreis auswirken. Um persönliche Kontakte und eine Vertrauensbasis mit Jugendlichen herzustellen, bedarf es allerdings eines hohen zeitlichen Aufwandes und einer permanenten Präsenz im Feld, die wir zwar im Rahmen unserer Dissertationen zu anderen Themenfeldern (Bauer 2010; Landolt 2011) aufbringen konnten und den so ermöglichten vertieften Einblick in die alltäglichen Lebenskontexte von jungen Menschen schätzen lernten. Aktuell kann dies im gleichen zeitlichen Umfang jedoch nicht eingelöst werden. Andererseits sind wir auch in die Weiterentwicklung des Projekts (z. B. Verbesserung des Auswahlverfahrens, Verdoppelung des Kursangebotes) und in die interne Kommunikation der aktiven Vereinsmitglieder eingebunden. Beides sind spannende Bereiche der wissenschaftlichen Be- 
gleitung eines solchen Bildungsprojektes. Jedoch kann eben nur eine punktuelle Unterstützung im Rahmen eines kleinen Zeitbudgets angeboten werden. Trotz dieser Einschränkung ist es dem Verein und uns als Bildungsgeographinnen wichtig das Projekt weiterhin wissenschaftlich zu untersuchen.

Interessante Themen, die es weiter zu vertiefen gilt, sehen wir z. B. in den internen Widersprüchen, für die der Verein „Go for it!” selbst steht. Denn auf der einen Seite engagiert er sich sehr für Kinder aus einkommensschwachen Familien mit und ohne Migrationshintergrund und bietet u.a. eine intensive Prüfungsvorbereitung und Probezeitbegleitung am Gymnasium an. Auf der anderen Seite wendet der Verein ebenfalls ein selektives Aufnahmeverfahren für den Kursbesuch an, weckt z. T. vielleicht überzogene Hoffnungen bei Kindern und Familien, die Aufnahmeprüfung aufgrund des Kursbesuchs sicher zu bestehen, und schließlich wird der Erfolg des jeweiligen Kurses von einigen Vereinsmitgliedern daran gemessen, wie viele Kinder die Aufnahmeprüfung geschafft haben, während andere Mitglieder/innen immer wieder die langfristigen Wirkungen des Kursbesuchs auf den Schulerfolg in der Sekundarstufe (entweder am Gymnasium oder in einer Sekundarschule) betonen. Der langfristige Effekt des Kursbesuches auf die weitere Bildungsbiographie und die Lern- und Leistungsbereitschaft der Jugendlichen ist jedoch nur schwer nachzuweisen - und das betrifft sowohl Jugendliche mit einem positiven, wie mit einem negativen Prüfungsergebnis bei der Aufnahmeprüfung fürs Gymnasium. Weil wir in diesem und anderen Themenbereichen großes Potenzial sehen, die Bildungsgeographien von Jugendlichen in der Schweiz genauer unter die Lupe zu nehmen, haben wir in einem ersten Schritt den Probezeitbesuch von „Go for it!"-Jugendlichen am Gymnasium im Rahmen einer Masterarbeit untersucht. Die Abschlussarbeit analysierte eingehend, wie das von „Go for it!” angebotene Tutorinnen- und Tutoren-Programm am Gymnasium von den Jugendlichen angenommen und genutzt wird bzw. welche Verbesserungen der Verein diesbezüglich vornehmen könnte (Publikation dazu in Vorbereitung). Ein nächster Schritt wird die Eingabe eines größeren Forschungsprojekts zum Bildungsmarkt an der Schwelle zum Gymnasium sein, das verschiedene Angebote und Fördermöglichkeiten für die Aufnahmeprüfung eingehend untersucht. Hierdurch sehen wir eine Möglichkeit das Engagement für „Go for it!” finanziell und personell längerfristig abzusichern und auszubauen. Besonders wertvoll erscheint uns, der allgemeinen Debatte zu mehr Chancengerechtigkeit im Schweizer Bildungssystem (u.a. Neuenschwander 2014; Becker 2010, 2013) eine empirische Studie zur Seite zu stellen, die explizit einen Fokus auf einen urban geprägten Kanton in der
Deutschschweiz legt. Dieses Fallbeispiel ist deswegen so interessant, weil gerade im lokalen Kontext zwei Extreme aufeinandertreffen: ein sehr hohes Bildungsniveau in der Bevölkerung (47\% mit Tertiärbildungsabschluss) und eine im (inter-)nationalen Vergleich sehr tiefe Gymnasialquote (ca. 20\%). Von der Bevölkerung steigt der Druck aufs Gymnasium, während von Seiten der Bildungspolitik ein regressiver Kurs entgegengehalten wird, weil die verfügbaren Plätze am Gymnasium eine Konstante darstellen, die nicht angetastet wird. Unter diesen Voraussetzungen bildete sich ein privater Bildungsmarkt, der eine kosten- und zeitintensive sowie erfolgsorientierte Prüfungsvorbereitung verkauft. Städtische Primarschulen bieten im Gegensatz dazu kostenlos eine Doppelstunde pro Woche an, in der nach Auskunft von Primarschullehrpersonen häufig alte Prüfungsaufgaben mit Schülerinnen und Schülern bearbeitet werden. Eine Untersuchung dieses Angebots auf ihre inhaltliche Qualität hin steht jedoch noch aus und wäre mit Sicherheit eine lohnende Aufgabe.

Der Verein „Go for it!” ist eine private Initiative, die einen Mittelweg zwischen diesen Extremen versucht. An diesem konkreten Fallbeispiel kann die ganze Spannbreite dieses Bildungsmarktsegments an unterschiedlichen Angeboten zur Prüfungsvorbereitung aufgezeigt werden - angefangen von den schulischen Vorbereitungskursen, den kommerziellen Lernstudios und Privat-Coaches, nicht-kommerziellen Initiativen wie "Go for it!", bis hin zu informellen privaten Netzwerken.

Dass wir bei der Analyse der Angebotsseite die Stimmen der Jugendlichen, die den Übertrittsprozess durchleben, nicht vergessen, ist uns ein wichtiges Anliegen einer sozial engagierten Jugend- und Bildungsgeographie mit Jugendlichen und für Jugendliche. Auch wenn die Namen der Jugendlichen im geplanten Forschungsvorhaben andere sein mögen, so haben Leyla, Serkan, Aylül, Denis und Ananthukama in ihren Familien und unter ihren Freundinnen und Freunden Hoffnungen geweckt und Perspektiven eröffnet, dass ambitionierte Jugendliche den Sprung ins Gymnasium schaffen können. Aus dem Wunsch „Ich will auch ans Gymi!“" (vgl. Kaufmann \& Engel 2019) wird dann eine selbstbewusste Haltung, die Kinder unabhängig vom Einkommen oder der Herkunft ihrer Eltern nachhaltig vertreten und für sich einfordern. Ein echter Schritt in Richtung mehr Chancengerechtigkeit im Schweizer Bildungssystem!

\section{Dank}

Die Autorin dankt der Initiative „Go for it!” für die großzügige Übernahme des Druckkostenzuschusses. 


\section{Literatur}

Baghdadi, N. \& Y. Riaño (2011): Negotiating Spaces of Participation: Experiences and Strategies of Skilled Immigrant Women Seeking Professional Integration. In: Oleksy, E. H., J. Hearn \& D. Golanska (Hrsg.): The Limits of Gendered Citizenship. Contexts and Complexeties. New York: Routledge. S. 176-196.

Bauer, I. (2010): Jugendgeographien. Ein subjekt- und handlungsorientierter Ansatz in Theorie und Praxis. LIT Verlag, Braunschweig.

Bauer, I. (2018): Framing, overflowing, and fuzzy logic in educational selection: Zurich as a case study. In: Geographica Helventica 73(1). S. 19-30.

Becker, R. (2010): Soziale Ungleichheit im Schweizer Bildungssystem und was man dagegen tun könnte. In: Neuenschwander, M. P. \& H.-U. Grunder (Hrsg.): Schulübergang und Selektion: Forschungsbefunde, Praxisbeispiele, Umsetzungsperspektiven. Rüegger, Zürich. S. 91-108.

Becker, R. (2013): Bildungsungleichheit und Gerechtigkeit in der Schweiz. In: Schweizerische Zeitschrift für Bildungswissenschaften 35(3). S. 405-413.

Breen, R., \& J. H. Goldthorpe (1997): Explaining Educational Differentials. Towards a Formal Rational Action Theory. In: Rationality and Society 9 (3). S. 275-305.

Fend, H. (2009): Chancengleichheit im Lebenslauf - Kurzund Langzeitwirkungen von Schulstrukturen. In: Fend, H., F. Berger \& U. Grob (Hrsg.): Lebensverläufe, Lebensbewältigung, Lebensglück. VS Verlag für Sozialwissenschaften, Wiesbaden. S. 37-72.

Kaufmann, C. \& K. Engelmann (2019): Ich will ans Gymi. Strategien, Tipps und Übungen für den Eintritt ins Langzeitgymnasium. Verlag der Zürcher Kantonalen Mittelstufenkonferenz, Zürich.

Gresch, C. (2012): Der Übergang in die Sekundarstufe I. Leistungsbeurteilung, Bildungsaspiration und rechtlicher
Kontext bei Kindern mit Migrationshintergrund. Springer VS, Wiesbaden.

Knittel, T., T. Schneidenbach \& S. Mohr (2015): JuvenirStudie 4.0: Zuviel Stress - zuviel Druck! Wie Schweizer Jugendliche mit Stress und Leistungsdruck umgehen. Jacobs-Stiftung, Zürich.

Landolt, S. (2011): Trinkräume und Treffpunkte Jugendlicher: Aushandlungsprozesse im öffentlichen Raum der Stadt Zürich. MNF, Universität Zürich.

Landolt, S. \& S. Thieme (2018): Highly skilled migrants entering the labour market: Experiences and strategies in the contested field of overqualification and skills mismatch. In: Geoforum 90. S. 36-44.

Moser, U., A. Buff, D. Angelone \& J. Hollenweger (2011): Nach sechs Jahren Primarschule. Deutsch, Mathematik und motivational-emotionales Befinden am Ende der 6. Klasse. Bildungsdirektion Kanton Zürich, Zürich.

Oelkers, J. (2008): Die Qualität der Schweizer Gymnasien. hip, Bern.

Neuenschwander, M. P. (2014): Selektion beim Übergang in die Sekundarstufe I und in den Arbeitsmarkt im Vergleich. In: Neuenschwander, M.P. (Hrsg.): Selektion in Schule und Arbeitsmarkt. Rüegger, Zürich. S. 63-98.

Schweizer Kommission für Bildungsforschung (SKBF) (2014): Bildungsbericht Schweiz. Schweizerische Koordinationsstelle für Bildungsforschung, Aarau.

Schweizer Kommission für Bildungsforschung (SKBF) (2018): Bildungsbericht Schweiz. Schweizerische Koordinationsstelle für Bildungsforschung, Aarau.

Schulte-Markwort, M. (2015): Burn out kids. Wie das Leistungsprinzip unsere Kinder überfordert. Pattloch, München.

Seiffge-Krenke, I. (2006): Nach PISA. Stress in der Schule und mit den Eltern. Bewältigungskompetenz deutscher Jugendlicher im internationalen Vergleich. Vandenhoeck \& Ruprecht, Göttingen. 